\title{
Aging continuous time random walks
}

\author{
Eli Barkai ${ }^{\mathrm{a})}$ \\ Department of Chemistry and Biochemistry, Notre Dame University, Notre Dame, Indiana 46556 \\ Yuan-Chung Cheng \\ Department of Chemistry, Massachusetts Institute of Technology, Cambridge, Massachusetts 02139
}

(Received 26 November 2002; accepted 17 January 2003)

\begin{abstract}
We investigate biased and nonbiased aging continuous time random walks (ACTRW), using fractal renewal theory. For example, a biased ACTRW process describes a Montroll-Weiss CTRW process which starts at time $-t_{a}$ and then at time $t=0$ a bias is added to the random walk (i.e., an external field is switched on). Statistical behaviors of the displacement of the random walker $\mathbf{r}=\mathbf{r}(t)$ $-\mathbf{r}(0)$ in the time interval $(0, t)$ are obtained, after aging the random walk in the time interval $\left(-t_{a}, 0\right)$. In ACTRW formalism, the Green function $P\left(\mathbf{r}, t_{a}, t\right)$ depends on the age of the random walk $t_{a}$ and the forward time $t$. We derive a generalized Montroll-Weiss equation, which yields an exact expression for the Fourier double Laplace transform of the ACTRW Green function. Asymptotic long times $t_{a}$ and $t$ behaviors of the Green function are shown to be related to the arc-sine distribution and Lévy stable laws. In the limit of $t \gg t_{a}$, we recover the standard nonequilibrium CTRW behaviors, while the important regimes $t \ll t_{a}$ and $t \simeq t_{a}$ exhibit interesting aging effects. Convergence of the ACTRW results towards the CTRW behavior, becomes extremely slow when the diffusion exponent becomes small. In the context of biased ACTRW, we investigate an aging Einstein relation. We briefly discuss aging in Scher-Montroll type of transport in disordered materials. (C) 2003 American Institute of Physics. [DOI: 10.1063/1.1559676]
\end{abstract}

\section{INTRODUCTION}

Diffusion and relaxation in strongly disordered systems exhibits in many cases anomalous behaviors. ${ }^{1-3}$ For example the diffusion of a test particle may become anomalous, namely the mean square displacement behaves like $\left\langle r^{2}\right\rangle$ $\sim t^{\alpha}$ and $\alpha \neq 1$. A random walk framework, widely applied to describe anomalous diffusion is the Montroll-Weiss continuous time random walk (CTRW) ${ }^{4-6}$ CTRWs are used to model many physical and chemical processes, for example: charge transport in disordered systems, ${ }^{7}$ protein folding dynamics, ${ }^{8-10}$ transport in low-dimensional chaotic systems, ${ }^{11-14}$ anomalous diffusion in a metallic supercooled liquid, ${ }^{15}$ the chemical reaction of $\mathrm{CO}$ binding to myoglobin, ${ }^{16}$ and blinking behavior of single quantum dots. ${ }^{17}$

Anomalous diffusion processes may exhibit aging, ${ }^{18-23}$ where vaguely speaking the age of the process controls the statistical properties of the random walk. Aging in diffusion processes yields an interesting insightful perspective on dynamics in disordered medium, and more generally is used as a tool to probe complex systems such as blinking quantum dots, ${ }^{24}$ spin glasses, Anderson insulators, and colloidal suspensions (see Ref. 25 for a brief review).

Monthus and Bouchaud ${ }^{26}$ introduced a CTRW framework, which exhibits aging behaviors. In what follows we call this generalized CTRW, aging continuous time random walk (ACTRW). The standard non-Markovian CTRW assumes that jumping transitions begin at time $t=0$, hence the CTRW describes a nonstationary process, where time homo-

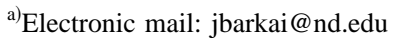

geneity is destroyed at time $t=0^{6}$. ACTRW describes a CTRW process which begun at time $-t_{a}$. When $t_{a}$ becomes long we show that a type of an equilibrium process emerges which still depends on time scale $t_{a}$. Thus generally ACTRW and CTRW exhibit different behaviors.

In this paper, we use Dynkin's ${ }^{27}$ and Feller's ${ }^{28}$ results on fractal renewal theory to investigate properties of biased and nonbiased ACTRW (and see also a very useful investigation of fractal renewal theory by Gordeche and $\mathrm{Luck}^{29}$ ). In particular, we obtain a generalized Montroll-Weiss equation yielding an exact expression for the Fourier double Laplace transform of the Green function. Asymptotic behaviors of ACTRW are shown to be related to the arc-sine distribution as well as to Lévy stable laws.

ACTRW might yield a phenomenological description of aging self-diffusion dynamics in glasses ${ }^{26}$ below the glass transition temperature $T_{c}$ and then $\alpha=T / T_{c}$ is temperature dependent (and see Refs. 30-34 for related work). Recently ${ }^{35}$ showed that ACTRW describes dynamics of an intermittent chaotic system. We note that other stochastic approaches to aging dynamics are based on a nonlinear diffusion equation $^{36}$ (however this equation does not always yield a normalized Green function), and a generalized Langevin equation. ${ }^{37,38}$

Investigation of ACTRW seems important for several reasons: (i) Consider a random walk process which starts at time $-t_{a}$, then at time $t=0$ we break the symmetry of the random walk by applying a uniform external field on the system. The response for any non-Markovian process to the external field will depend both on the forward time $t$ and the aging period $t_{a}$. Since the response depends on two times it 
contains more information than the standard treatment of CTRW theory which assumes $t_{a}=0$. It is not known yet if ACTRW can be used to describe transport in complex systems when aging conditions are applied. However due to the large number of application of the nonequilibrium CTRW we may expect that also ACTRW has a certain domain of validity in the physical world, when aging initial conditions are satisfied. (ii) Consider now nonbiased random walks. In some physical situations we may not know what is $t_{a}$, since particles in a solution do not carry a birth certificate. Thus if we make an observation of location of random walkers at time $t=0$ (assume we cannot identify wether a transition occurred at time $t=0^{-}$) and then ask for example what is the mean square displacement of such particles, the answer according to ACTRW depends on how old is the random walk process. Nonequilibrium CTRW yields an answer which is independent of age of the processes. We see that anomalous behavior of a particle is very sensitive to the measurement procedure. If one can identify start of measurement $t=0$ with a jumping event nonequilibrium CTRW is a more suitable framework, while ACTRW is more suitable for an ongoing process. (iii) Recently fractional kinetic equations $^{2,39-41}$ where suggested as a stochastic tool to investigate anomalous transport, these equations are based on nonequilibrium CTRW and thus they are not suited to describe an ongoing process. ${ }^{42,43}$ Our work points out to the limitations of these equations, and yields a way to correct their solution when aging conditions are included.

This paper is organized as follows: In Sec. II CTRW and ACTRW are introduced. We then derive an exact expression for the Fourier-double Laplace transform of the ACTRW Green function $P\left(\mathbf{r}, t_{a}, t\right)$, thus generalizing the MontrollWeiss equation to include the effect of the age of the random walk (see Sec. III). In Sec. IV we derive asymptotic behaviors of the Green function $P\left(\mathbf{r}, t_{a}, t\right)$ which are analyzed in detail. We then consider biased ACTRWs (Sec. V) and discuss an aging Einstein relation. Finally we briefly consider aging in Scher-Montroll type of transport as a possible application of this work. Note that a small part of our results was reported in Ref. 35, in the context of aging in chaotic transport.

\section{CTRW AND ACTRW}

One of the best well-known random walk models is the CTRW introduced by Montroll and Weiss. ${ }^{4}$ It describes a large class of random walks, both normal and anomalous and can be described as follows: Suppose a particle performs a random walk in such a way that the individual jump $\mathbf{r}$ in space is governed by a probability density function (PDF) $f(\mathbf{r})$, and that all jump vectors are independent and identically distributed. The characteristic function of the position of the particle relative to the origin after $n$ jumps is $f^{n}(\mathbf{k})$, where $f(\mathbf{k})$ is the Fourier transform of $f(\mathbf{r})$. Unlike discrete time random walks, the CTRW describes a situation where the waiting time $t$ between jumps is not a constant. Rather, the waiting time is governed by the PDF $\psi(t)$ and all waiting times are mutually independent and identically distributed. Thus, number of jumps $n$ is a random variable.

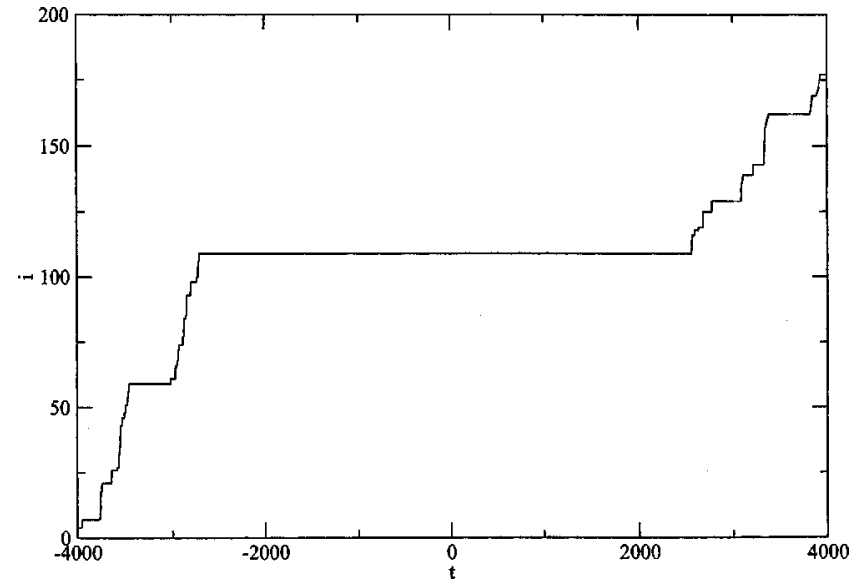

FIG. 1. Number of jumps $i$ in a renewal process with $\psi(t)=\pi^{-1} t^{-1 / 2}(1$ $+t)^{-1}$, i.e., $\alpha=1 / 2$. The process starts at $t=-t_{a}$ with $t_{a}=4000$. Observation of the process begins at time $t=0 . t_{1}$ is the time elapsing between $t$ $=0$ and first jump event in the forward time interval $(0, t)$, in the figure. $t_{1}=2553$.

Let $P_{\mathrm{MW}}(\mathbf{r}, t)$ be the Green function of the CTRW, the Montroll-Weiss equation yields this function in FourierLaplace $(\mathbf{k}, u)$ space,

$$
P_{\mathrm{MW}}(\mathbf{k}, u)=\frac{1-\psi(u)}{u} \frac{1}{1-f(\mathbf{k}) \psi(u)} .
$$

All along this work we will use the convention that the arguments in the parentheses define the space we are working in, thus $\psi(u)$ is the Laplace transform of $\psi(t)$. Properties of $P_{\mathrm{MW}}(\mathbf{r}, t)$ based on the Fourier-Laplace inversion of Eq. (1) are well investigated, see Refs. 5 and 40 and References therein. In particular, it is well known that asymptotic behavior of $P_{\mathrm{MW}}(\mathbf{r}, t)$ depends on the long time behavior of $\psi(t)$. Two classes of processes are usually considered. The first is the case when all moments of $\psi(t)$ are finite, the second class is the case where $\psi(t)$ is momentless, corresponding to a situation where $\psi(t) \propto t^{-(1+\alpha)}$ and $0<\alpha<1$.

An important assumption made in the derivation of Eq. (1) is that the random walk begun at time $t=0$. More precisely, it is assumed that the PDF of the first waiting time, i.e., the time elapsing between start of the process at $t=0$ and the first jump event is $\psi(t)$. Thus the Montroll-Weiss CTRW approach describes a particular choice of initial conditions, called nonequilibrium initial conditions. The limitation of CTRW theory to a very particular choice of initial conditions, was an issue for debates in the early 1970s. ${ }^{44}$

Monthus and Bouchaud ${ }^{26}$ introduced a CTRW for an ongoing process, where the random walk process is assumed to start at some time $t=-t_{a}$, long before start of observation at time $t=0$. In Fig. 1 a stochastic realization of number of jumps in such a process is shown. For such a random walk the Green function is denoted with $P\left(\mathbf{r}, t_{a}, t\right)$ and $\mathbf{r}$ is the displacement in the time interval $(0, t)$. Using scaling analysis, ${ }^{26}$ have investigated basic properties of this random walk, mainly the behavior of the Fourier transform of the Green function. For $\alpha<1$ the random walk exhibits interesting aging effects, hence as mentioned we call it ACTRW. 
There exist several methods to investigate aging. One method is to start a dynamical process at time $t=-t_{a}$, then at time $t=0$ add a small perturbation to the system. One eventually measures the response at some time $t>0$. This type of aging is investigated here in the context of biased ACTRW. Alternatively one can measure displacement of a random walker during a time interval $(0, t)$, after aging the process in the interval $\left(-t_{a}, 0\right)$ (i.e., now no external field is switched on at time $t=0$ ). We investigate this behavior in the context of nonbiased ACTRW and will show later that under certain conditions the two approaches are related through a generalized aging Einstein relation. A system exhibits aging if its dynamical properties depend on $t$ and $t_{a}$ even in the limit when both are long. Of course many systems do not exhibit aging, namely when $t>\tau$, where $\tau$ is a characteristic time scale of the problem, dynamical properties of the process are independent of the aging time $t_{a}$.

\section{ACTRW: GENERALIZED MONTROLL-WEISS EQUATION}

The ACTRW describes the following process, a particle is trapped on the origin for time $t_{1}$, it then jumps to $\mathbf{r}_{1}$, the particle is then trapped on $\mathbf{r}_{1}$ for time $t_{2}$, and then it jumps to a new location; the process is then renewed. Thus, the ACTRW process is characterized by a set of waiting times $\left\{t_{1}, \ldots, t_{n}, \ldots\right\}$ and displacements $\left\{\mathbf{r}_{1}, \ldots, \mathbf{r}_{\mathbf{n}}, \ldots\right\}$. The time elapsing between start of observation at $t=0$, and the first jump event is denoted by $t_{1}$. Here we denote the PDF of the first waiting time $t_{1}$ with $h_{t_{a}}\left(t_{1}\right)$. In ACTRW the random walk started at $t=-t_{a}$, before the start of observation at $t$ $=0$, therefore $h_{t_{a}}\left(t_{1}\right)$ depends on age of the process $t_{a}$. The waiting times $\left\{t_{n}\right\}$, with $n>1$ are independent and identically distributed with a common probability density $\psi(t)$. The jump length $\left\{\mathbf{r}_{\mathbf{1}}, \ldots, \mathbf{r}_{\mathbf{n}}, \ldots\right\}$, are independent identically distributed random variables, described by the probability density $f(\mathbf{r})$.

In contrast, in the Montroll-Weiss nonequilibrium CTRW, the age of the process is zero $t_{a}=0$. And, for that case $h_{t_{a}}\left(t_{1}\right)=\psi\left(t_{1}\right)$.

Recently, Gordeche and Luck $^{29}$ investigated statistical properties of fractal renewal processes, among other things they obtain $h_{t_{a}}\left(t_{1}\right)$. Let $h_{s}(u)$ be the double Laplace transform of $h_{t_{a}}\left(t_{1}\right)$

$$
h_{s}(u)=\int_{0}^{\infty} \mathrm{d} t_{1} \int_{0}^{\infty} \mathrm{d} t_{a} h_{t_{a}}\left(t_{1}\right) e^{-t_{a} s-t_{1} u},
$$

then according to Ref. 29,

$$
h_{s}(u)=\frac{1}{1-\psi(s)} \frac{\psi(s)-\psi(u)}{u-s} .
$$

In the Appendix we rederive Eq. (3) using a method which slightly differs from the one used in Ref. 29.

Two types of behaviors are found for $h_{t_{a}}\left(t_{1}\right)$. The first case corresponds to a situation when average waiting time $\langle t\rangle=\int_{0}^{\infty} t \psi(t) \mathrm{d} t$ is finite, and then in the long aging time limit one obtains ${ }^{28}$

$$
\lim _{t_{a} \rightarrow \infty} h_{t_{a}}\left(t_{1}\right)=\frac{\int_{0}^{t_{1}} \psi(t) \mathrm{d} t}{\langle t\rangle} .
$$

This type of initial condition is called equilibrium initial condition, it was investigated previously in the context of CTRW and related models. ${ }^{4-46}$ Feller $^{28}$ derived Eq. (4) from the requirement that the renewal process be stationary. Here we will mainly consider the second case corresponding to a power law waiting time PDF,

$$
\psi(t) \propto t^{-(1+\alpha)} \quad \text { with } 0<\alpha<1,
$$

when $t$ is long. In Laplace $t \rightarrow u$ space Eq. (5) reads

$$
\psi(u) \sim 1-A u^{\alpha},
$$

where $u$ is small, and $A$ is a positive parameter. ${ }^{47}$ For example the one sided Lévy PDFs whose Laplace pair is $\psi(u)=\exp \left(-A u^{\alpha}\right)$, or $\psi(u)=1 /\left(1+A u^{\alpha}\right)$ discussed below, belong to the class of functions described by Eqs. (5) and (6). For this case $\langle t\rangle=\infty$ and Eq. (4) is not valid. According to Dynkin's limit theorem, ${ }^{27,28}$ in the limit of long aging times, these kind of probability densities yield

$$
h_{t_{a}}\left(t_{1}\right) \sim \frac{\sin (\pi \alpha)}{\pi} \frac{t_{a}^{\alpha}}{t_{1}^{\alpha}\left(t_{1}+t_{a}\right)} .
$$

Note that this expression is independent of the exact form of $\psi(t)$, except for the exponent $\alpha$. When $\alpha \rightarrow 1$ the mass of the PDF $h_{t_{a}}\left(t_{1}\right)$ is concentrated in the vicinity of $t_{1} \rightarrow 0$, as expected from a normal process. Equation (7) shows that as age of the process becomes older, we have to wait longer for first jumping event to occur. In a physical process, this may correspond to a particle in a disordered system which searches for a local energy minima in time interval $\left(-t_{a}, 0\right)$. In this case the longer the search takes place the deeper the minima found, hence in statistical sense the release time becomes longer as the process is older. In what follows, we will also use the double Laplace transform of Eq. (7):

$$
h_{s}(u) \sim \frac{u^{\alpha}-s^{\alpha}}{s^{\alpha}(u-s)} .
$$

This equation can be derived by inserting the small $u$ and $s$ expansion of $\psi(u)$ and $\psi(s)$ given in Eq. (6), in Eq. (3). Feller $^{28}$ discusses the relation of Eq. (7) to the so-called arcsine distribution (see the Appendix for some details).

Let $P\left(\mathbf{r}, t_{a}, t\right)$ be the Green function of the random walker, where as mentioned

$$
\mathbf{r} \equiv \mathbf{r}(t)-\mathbf{r}(0)
$$

is the displacement in the time interval $(0, t)$. Hence, clearly initially $\mathbf{r}=0$ at time $t=0$. Let (i) $p_{n}\left(t_{a}, t\right)$ be the probability of making $n$ steps in the time interval $(0, t)$ and (ii) $P(\mathbf{k}, s, u)$ be the double-Laplace-Fourier transform $\left(\mathbf{r} \rightarrow \mathbf{k}, t_{a} \rightarrow s, t\right.$ $\rightarrow u)$ of $P\left(\mathbf{r}, t_{a}, t\right)$. Then

$$
P(\mathbf{k}, s, u)=\sum_{n=0}^{\infty} p_{n}(s, u) f^{n}(\mathbf{k}),
$$


where $p_{n}(s, u)$ is the double Laplace-transform of $p_{n}\left(t_{a}, t\right)$. As mentioned, $f^{n}(\mathbf{k})$ in Eq. (10) is the characteristic function of a random walk with exactly $n$ steps. Using the convolution theorem of Laplace transform we obtain

$$
p_{n}(s, u)= \begin{cases}\frac{1-s h_{s}(u)}{s u}, & n=0, \\ h_{s}(u) \psi^{n-1}(u) \frac{1-\psi(u)}{u}, & n \geqslant 1 .\end{cases}
$$

Hence inserting Eq. (11) in Eq. (10), using Eq. (3), and summing over $n$, we find the exact result

$$
P(\mathbf{k}, s, u)=\frac{1}{s u}+\frac{[\psi(u)-\psi(s)][1-f(\mathbf{k})]}{u(u-s)[1-\psi(s)][1-\psi(u) f(\mathbf{k})]} .
$$

Equation (12) is a generalization of the Montroll-Weiss equation (1) for ACTRW. Note that $P(\mathbf{k}=0, s, u)=1 /(s u)$ as expected from the normalization condition.

It is useful to rewrite Eq. (12) in terms of the MontrollWeiss Eq. (1), and $p_{0}(s, u)$ in the first line of Eq. (11):

$$
P(\mathbf{k}, s, u)=p_{0}(s, u)+h_{s}(u) f(\mathbf{k}) P_{\mathrm{MW}}(\mathbf{k}, u) .
$$

The first term on the right-hand side of this equation, describes random walks where the particle does not leave the origin (i.e., $n=0$ ). The second term describes random walks where number of steps is greater than zero, it is given in terms of a convolution of $h_{s}(u) f(\mathbf{k})$ with the MontrollWeiss equation. This is expected since the only difference between ACTRW and the nonequilibrium CTRW, is the first waiting time distribution.

If the process is Poissonian, $\psi(t)=\exp (-t)$, the Green function $P\left(\mathbf{r}, t, t_{a}\right)$ is independent of the age of the random walk $t_{a}$. To show this we insert

$$
\psi(u)=\frac{1}{1+u}, \quad \psi(s)=\frac{1}{1+s}
$$

in Eq. (12) and find

$$
P(\mathbf{k}, s, u)=\frac{1}{s} \frac{1}{u+1-f(\mathbf{k})} .
$$

Inverting to the double time domain

$$
P\left(\mathbf{k}, t_{a}, t\right)=e^{-[1-f(\mathbf{k})] t} .
$$

This result is independent of $t_{a}$ as expected from a Markovian process. Assume that $f(\mathbf{k})=1-m_{\mu}|\mathbf{k}|^{\mu}+\cdots$ for small values of $\mathbf{k}$ and $\mu \leqslant 2$, implying that the random walks is nonbiased. In the long time limit $P\left(\mathbf{k}, t_{a}, t\right)$ $\sim \exp \left(-m_{\mu}|k|^{\mu} t\right)$, and either a Lévy behavior $(\mu<2)$ or a Gaussian behavior $(\mu=2)$ is found, as expected from the Gauss-Lévy central limit theorem. ${ }^{28}$ In what follows, we investigate cases when this standard behavior does not hold.

\section{ASYMPTOTIC BEHAVIORS: NONBIASED ACTRW}

Let us now consider basic properties of nonbiased ACTRWs. While Eq. (12) is valid for a large class of random walks, including Lévy flights $(\mu<2)$, we will assume that variance of $f(\mathbf{r})$ is finite $(\mu=2)$. Special emphasis will be given to the case when $\psi(t)$ is momentless $\alpha<1$, since this regime exhibits interesting aging behaviors.

\section{A. Mean square displacement}

By differentiating Eq. (12) with respect to $\mathbf{k}$ and setting $\mathbf{k}=0$, we obtain the moments of the random walk in a standard way. Assuming a nonbiased symmetrical random walk, we obtain

$$
\left\langle r^{2}(s, u)\right\rangle=\frac{h_{s}(u) m_{2}}{u[1-\psi(u)]},
$$

where $m_{2}=\int r^{2} f(|\mathbf{r}|) \mathrm{d} \mathbf{r}$ is assumed to be finite. We consider power law waiting time PDFs as in Eqs. (5) and (6), in the limit where both $u$ and $s$ are small, their ratio being arbitrary, we find

$$
\left\langle r^{2}(s, u)\right\rangle \sim \frac{\left(u^{\alpha}-s^{\alpha}\right) m_{2}}{s^{\alpha}(u-s) A u^{1+\alpha}} .
$$

As shown below one can invert this equation exactly to the double time domain. However it is instructive to consider two limits first. If $u \ll s$, corresponding to $t \gg t_{a}$, we have

$$
\left\langle r^{2}(s, u)\right\rangle \sim \frac{u^{-1-\alpha} m_{2}}{A s} .
$$

While for $s \ll u$, corresponding to $t_{a} \gg t$, we have

$$
\left\langle r^{2}(s, u)\right\rangle \sim \frac{m_{2}}{A u^{2} s^{\alpha}} .
$$

Inverting Eq. (19) and Eq. (20), we obtain

$$
\left\langle r^{2}\left(t_{a}, t\right)\right\rangle \sim \begin{cases}\frac{m_{2} t^{\alpha}}{A \Gamma(1+\alpha)}, & t \gg t_{a} \\ \frac{m_{2} t t_{a}^{\alpha-1}}{A \Gamma(\alpha)}, & t \ll t_{a} .\end{cases}
$$

This result is valid provided that both $t, t_{a} \gg A^{1 / \alpha}$. In the limit $t \gg t_{a}$ we recover standard behavior found in nonequilibrium CTRW. ${ }^{5}$ In the aging regime, $t \ll t_{a}$ we find an interesting behavior. Independent of the exponent $\alpha$, the mean square displacement increases linearly with respect to the forward time $t$, as found in normal diffusion processes. In addition, the diffusion is slowed down as the age of the random walk $t_{a}$ is increased. This behavior is expected, due to statistically longer release times, from the initial position of the particle, as the age of the random walk is increased.

We now consider a specific choice of waiting time distribution,

$$
\psi(u)=\frac{1}{1+A u^{\alpha}},
$$

corresponding to Ref. 48 ,

$$
\psi(t)=\frac{t^{\alpha-1}}{A} E_{\alpha, \alpha}\left(-\frac{t^{\alpha}}{A}\right),
$$

where $E_{\alpha, \alpha}(x)$ is the generalized Mittag-Leffler function. ${ }^{49}$ Inserting Eq. (22) in Eq. (17) we have 


$$
\left\langle r^{2}(s, u)\right\rangle=\frac{m_{2}}{A} \frac{u^{\alpha}-s^{\alpha}}{s^{\alpha}(u-s)} \frac{1}{u^{1+\alpha}} .
$$

Hence for this choice of waiting times, Eq. (18) is exact and not limited to the asymptotic regime. Inverting to the time domain using Eq. (7) we find

$$
\left\langle r^{2}\left(t_{a}, t\right)\right\rangle=\frac{m_{2}}{A} \frac{\sin (\pi \alpha)}{\pi} \frac{1}{t_{a}\left(\frac{t}{t_{a}}\right)^{\alpha}\left(1+\frac{t}{t_{a}}\right)} \otimes \frac{t^{\alpha}}{\Gamma(1+\alpha)},
$$

where $\otimes$ is the Laplace convolution operator with respect to the forward time $t$. We rewrite Eq. (25) as

$$
\left\langle r^{2}\left(t_{a}, t\right)\right\rangle=t_{a}^{\alpha} \frac{m_{2}}{A \Gamma(1+\alpha)} \frac{\sin (\pi \alpha)}{\pi} \int_{0}^{t / t_{a}} \frac{\left(t / t_{a}-y\right)^{\alpha}}{y^{\alpha}(1+y)} \mathrm{d} y .
$$

The solution of the integral is readily obtained, we find

$$
\left\langle r^{2}\left(t_{a}, t\right)\right\rangle=\frac{m_{2}}{A} \frac{1}{\Gamma(1+\alpha)}\left[\left(t+t_{a}\right)^{\alpha}-t_{a}^{\alpha}\right] .
$$

The right-hand side of Eq. (27) describes the long time $t$, long time $t_{a}$, behavior of a large class of random walks with waiting time PDF satisfying $\psi(t) \propto t^{-(1+\alpha)}$ [i.e., since the right-hand side of Eq. (27) is the double inverse Laplace transform of Eq. (18)]. Note that if $\alpha=1$ in Eq. (27), the random walk does not exhibit aging, this is expected since for $\alpha=1$ the generalized Mittag-Leffler function is an exponential and then the process has no memory.

\section{B. Green function}

In this section we investigate asymptotic properties of the Green function $P\left(\mathbf{r}, t_{a}, t\right)$, by considering the continuum approximation of Eq. (12). This approximation is expected to work in the limit where both the forward time $t$ and the aging time $t_{a}$ are long. A proof of the validity of this approach, is given in Sec. IV D, for the one-dimensional ACTRW. We assume a symmetric random walk, hence for small $|\mathbf{k}|$, the following expansion is valid:

$$
f(\mathbf{k}) \sim 1-\frac{1}{2}|\mathbf{k}|^{2} \frac{m_{2}}{d}
$$

where $d$ is the dimensionality of the problem. We also use the small Laplace variable $u$ expansion $\psi(u) \sim 1-A u^{\alpha}$ in Eq. (6). Inserting these expansions in Eq. (12), we obtain

$$
P(\mathbf{k}, u, s) \sim \frac{s^{\alpha} u-s u^{\alpha}}{s^{\alpha+1} u(u-s)}+\frac{\left(u^{\alpha}-s^{\alpha}\right)}{s^{\alpha}(u-s)} \frac{A u^{\alpha-1}}{A u^{\alpha}+|\mathbf{k}|^{2} \frac{m_{2}}{2 d}} .
$$

For convenience, and without loss of generality, we choose now to work in units where $A=1$ and $m_{2} /(2 d)=1$.

Inverting Eq. (29) to the double time $\left(t_{a}, t\right)$-real space $\mathbf{r}$ domain, we find

$$
P\left(\mathbf{r}, t_{a}, t\right) \sim p_{0}\left(t_{a}, t\right) \delta(\mathbf{r})+\frac{\sin (\pi \alpha)}{\pi} \frac{1}{t_{a}\left(\frac{t}{t_{a}}\right)^{\alpha}\left(1+\frac{t}{t_{a}}\right)}
$$

$$
\otimes P_{\mathrm{AMW}}(\mathbf{r}, t)
$$

where $\otimes$ in Eq. (30) is the Laplace convolution operator with respect to the forward time $t$, and in this limit

$$
p_{0}\left(t_{a}, t\right) \sim \frac{\sin (\pi \alpha)}{\pi} \int_{t / t_{a}}^{\infty} \frac{\mathrm{d} x}{x^{\alpha}(1+x)} .
$$

In Eq. (30) $P_{\mathrm{AMW}}(\mathbf{r}, t)$ is the long time solution of the Montroll-Weiss equation, i.e., the Green function of the fractional diffusion equation, ${ }^{2}$

$$
P_{\text {AMW }}(\mathbf{r}, t) \equiv \mathcal{L}^{-1} \mathcal{F}^{-1}\left\{\frac{u^{\alpha-1}}{u^{\alpha}+|\mathbf{k}|^{2}}\right\},
$$

where $\mathcal{L}^{-1} \mathcal{F}^{-1}$ is the inverse Laplace $u \rightarrow t$ inverse Fourier $\mathbf{k} \rightarrow \mathbf{r}$ operator.

The Green function Eq. (30), is a sum of two terms. The first term on the right-hand side of Eq. (30) is a singular term [i.e., the $\delta(\mathbf{r})$ term]. This term corresponds to random walks where number of steps in time interval $(0, t)$ is zero. Unless $t \gg t_{a}$, this term cannot be neglected, since without it the Green function in Eq. (30) is not normalized. Thus ACTRW exhibits a behavior different than ordinary CTRWs, where realizations of random walks where number of steps is zero do not contribute to the asymptotic behavior.

In one dimension, we have

$$
P_{\mathrm{AMW}}(r, t)=\frac{t}{\alpha|x|^{1+2 / \alpha}} l_{\alpha / 2}\left(\frac{t}{|x|^{2 / \alpha}}\right),
$$

where $l_{\alpha / 2}(t)$ is the one sided Lévy stable PDF, whose Laplace pair is $\exp \left(-u^{\alpha / 2}\right)$. Hence the Green function solution of the ACTRW is

$$
\begin{gathered}
P\left(x, t_{a}, t\right) \sim p_{0}\left(t_{a}, t\right) \delta(x)+\frac{\sin (\pi \alpha)}{\pi} \frac{1}{t_{a}\left(\frac{t}{t_{a}}\right)^{\alpha}\left(1+\frac{t}{t_{a}}\right)} \\
\otimes \frac{t|x|^{-(1+2 / \alpha)}}{\alpha 2^{1 / \alpha}} l_{\alpha / 2}\left(\frac{t|x|^{-(2 / \alpha)}}{2^{1 / \alpha}}\right) .
\end{gathered}
$$

Scaling Eq. (34) with $\tau \equiv t / t_{a}$ and $q \equiv|x| / t^{\alpha / 2}$ we obtain a scaled form of the Green function

$$
\begin{aligned}
P\left(x, t_{a}, t\right) \sim & p_{0}\left(t_{a}, t\right) \delta(x)+\frac{\sin (\pi \alpha)}{\pi \alpha} t^{-\alpha / 2} \frac{1}{\tau q^{1+2 / \alpha}} \\
& \times \int_{0}^{\tau} \mathrm{d} \tau^{\prime} \frac{\tau^{\prime}}{\left(\tau-\tau^{\prime}\right)^{\alpha}\left(1+\tau-\tau^{\prime}\right)} l_{\alpha / 2}\left(\frac{\tau^{\prime}}{q^{2 / \alpha} \tau}\right) .
\end{aligned}
$$

Below we analyze this expression in some detail.

In $d$ space dimensions we have $\mathrm{e}^{50}$

$$
\begin{aligned}
P_{\mathrm{AMW}}(r, t)= & \alpha^{-1} \pi^{-d / 2} r^{-d} \\
& \times H_{12}^{20}\left(2^{-2 / \alpha} r^{2 / \alpha} t^{-1} \mid \begin{array}{c}
(1,1) \\
(d / 2,1 / \alpha),(1,1 / \alpha)
\end{array}\right),
\end{aligned}
$$

Besides the $d=1$ case, the Fox function solution $H_{12}^{20}$ is not generally tabulated, hence this solution is rather formal, though asymptotic behaviors of Eq. (36) are well investigated. ${ }^{40,50}$ A practical method of obtaining the solution of $P_{A M W}(r, t)$ Eq. (36), using the inverse Lévy transform, is given in Ref. 40. Using this method, we find the integral representation of the aging Green function in $d$ dimension 


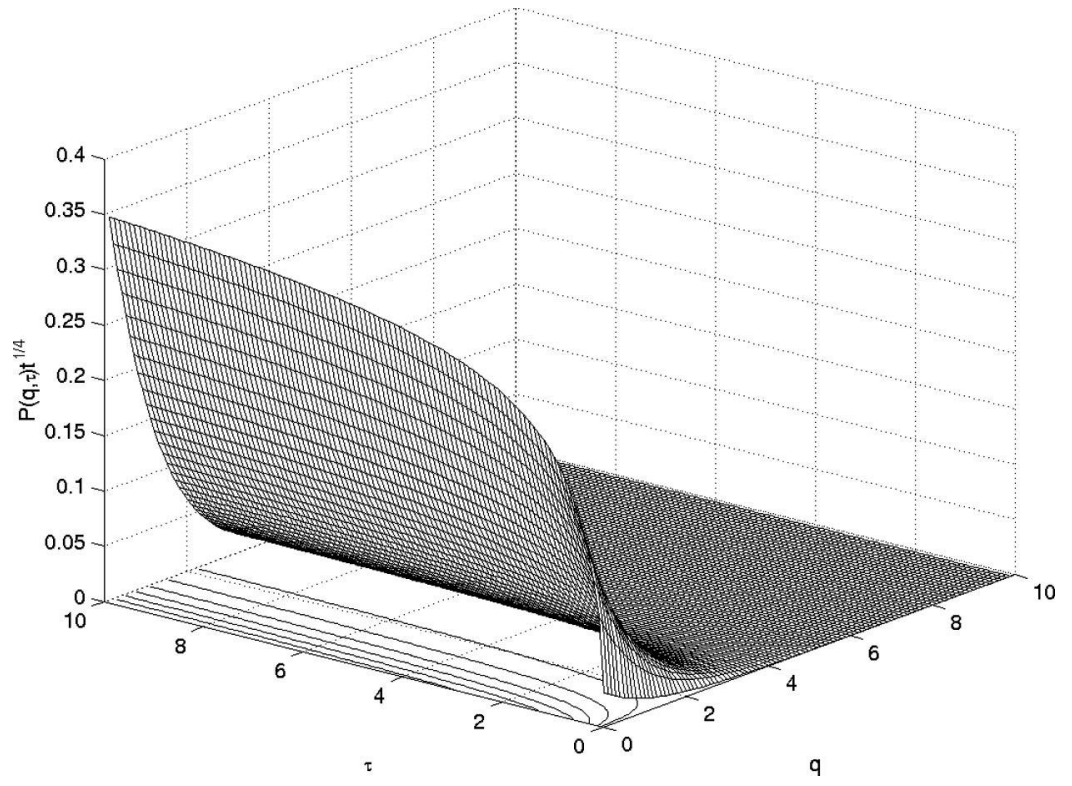

FIG. 2. Three-dimensional plot of the scaled nonsingular Green function vs $\tau=t / t_{a}$ and $q=|x| / t^{\alpha / 2}$ for $\alpha$ $=1 / 2$ and one dimension. Notice a smooth transition from aging behavior $\tau \ll 1$ to Montroll-Weiss CTRW behavior found when $\tau \gg 1$.

$$
\begin{aligned}
P\left(r, t_{a}, t\right) \sim & p_{0}\left(t_{a}, t\right) \delta(\mathbf{r})+\frac{1}{\tau} \frac{\sin (\pi \alpha)}{2^{d} \pi^{d / 2+1} \alpha} t^{-\alpha d / 2} \\
& \times \int_{0}^{\infty} \mathrm{d} s \int_{0}^{\tau} \mathrm{d} \tau^{\prime} \frac{\left(\tau-\tau^{\prime}\right)}{s^{1+1 / \alpha+d / 2}} l\left[\frac{\left(\tau-\tau^{\prime}\right)}{\tau s^{1 / \alpha}}\right] \\
& \times \frac{e^{-q^{2} /(4 s)}}{\tau^{\prime \alpha}\left(1+\tau^{\prime}\right)},
\end{aligned}
$$

where $q=r / t^{\alpha / 2}$ and $\tau=t / t_{a}$. Similar to the one-dimensional case, this solution shows the precise relation between ACTRWs, Lévy stable laws and Dynkin's limit theorem.

To conclude, Eq. (30) shows that the asymptotic solution of ACTRW is a sum of two terms. The first is a singular term, and the second is a convolution of the distribution of the first waiting time and the asymptotic Green function of the non equilibrium CTRW.

\section{Graphic examples}

In order to better understand the asymptotic behavior of the ACTRW, we perform numerical calculations to obtain the nonsingular part of the Green function for different values of $\alpha$ in one dimension. The one sided Lévy stable probability density in Eq. (33) was obtained using a numerical inverse Laplace transformation method. ${ }^{51,52}$ The calculated PDF was then used to evaluate the convolution integral numerically to obtain the nonsingular part of the Green function according to Eq. (35). For mathematical details on one sided Lévy stable laws, see Appendix in Ref. 40, and references therein.

In Figs. 2-5, we present the calculated nonsingular part of the Green function for different $\alpha$. Figure 2 shows a threedimensional plot of the scaled nonsingular Green function, $P(q, \tau) \cdot t^{\alpha / 2}$, versus scaling variables, $\tau=t / t_{a}$ and $q$ $=|x| / t^{\alpha / 2}$, for $\alpha=1 / 2$. A smooth transition from the aging

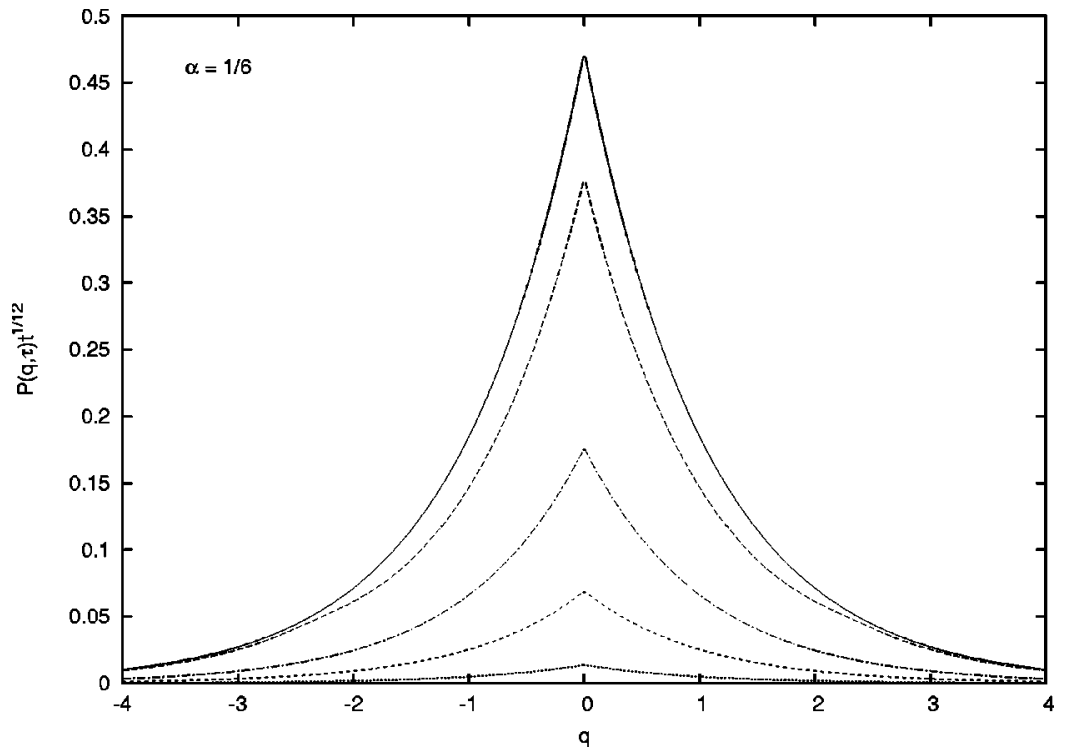

FIG. 3. The scaled nonsingular Green function vs $q$ $=|x| / t^{\alpha / 2}$ for $\alpha=1 / 6$ and for five different $\tau=t / t_{a}: \tau$ $=0.1$ (dotted), $\tau=1$ (dashed), $\tau=10$ (dot-dashed), $\tau$ $=10000$ (dashed), and $\tau=\infty$ (solid). The solid curve is the asymptotic behavior of the nonequilibrium CTRW. Notice the non-Gaussian shape of the Green function and the slow convergence towards the nonaging CTRW behavior (the solid line), compared with the cases $\alpha$ $=1 / 2$ and $\alpha=5 / 6$ shown below. 


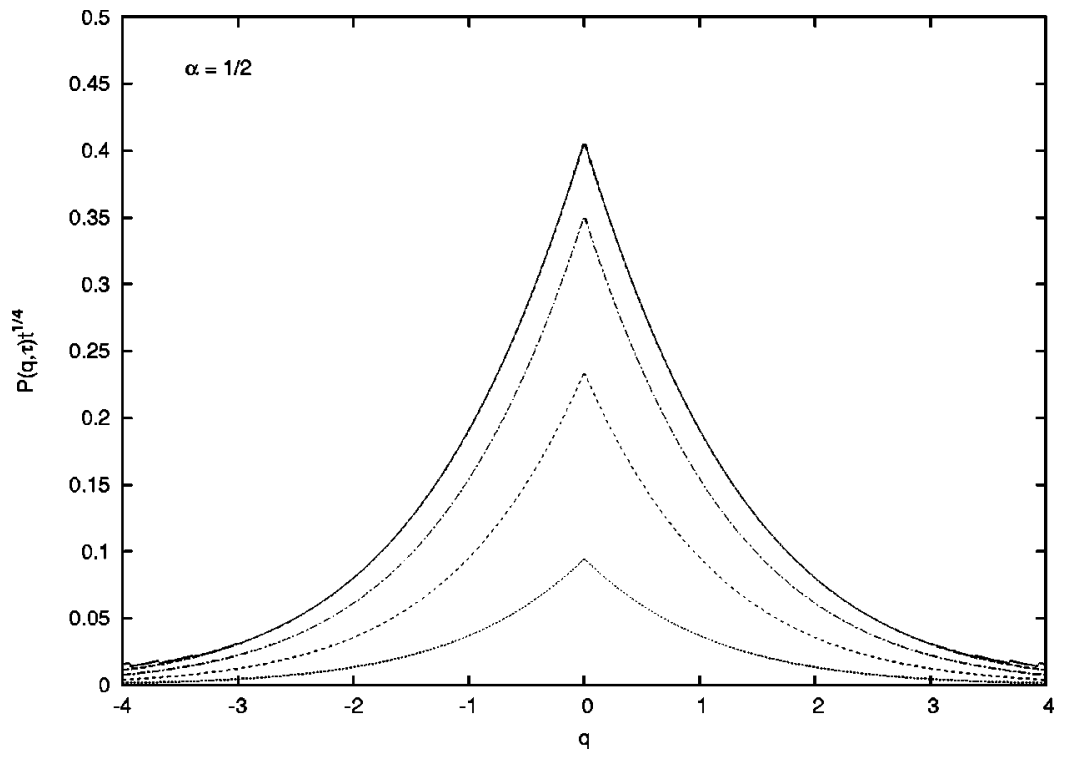

FIG. 4. Same as Fig. 3 for $\alpha=1 / 2$.

behavior when $\tau \ll 1$ to the nonequilibrium CTRW behavior when $\tau \gg 1$ can be clearly seen in this figure, where the time ratio $\tau=t / t_{a}$ is changed continuously. In addition, we observe a monotonic increase of the nonsingular Green function as the scaled time $\tau$ increases. Note that as the scaled $\tau$ is increased the singular term is decreasing, hence we may think of this aging process, as if the singular part of the Green function, is feeding the nonsingular part.

Figures 3-5 show the scaled nonsingular Green function versus $q=|x| / t^{\alpha / 2}$ for $\alpha=1 / 6, \alpha=1 / 2$, and $\alpha=5 / 6$, respectively. In each figure, the scaled Green functions at several different $\tau$ 's are shown. A few general features can be seen in these figures. First, the Green function is clearly nonGaussian for all cases, as we expected. A comparison between the shape of the Green function for $\alpha=1 / 6$ in Fig. 3 and for $\alpha=5 / 6$ in Fig. 5 clearly demonstrates that the deviations from Gaussian behavior are stronger for smaller $\alpha$. In the limit of $\alpha \rightarrow 1$ (not shown), we obtain a Gaussian Green function. Second, for $t \gg t_{a}$ (i.e., $\tau \gg 1$ ), we recover the usual nonequilibrium CTRW behavior shown as the solid curves in Figs. 3-5. Finally, as $\alpha$ becomes small, the convergence towards the nonaging behavior when $t / t_{a}=\infty$ becomes extremely slow. For example, the case for $\alpha=1 / 6$ in Fig. 3 shows a significant deviation from the nonequilibrium CTRW behavior (solid line) when $t / t_{a}=10000$. As a result, the Green function exhibits aging behavior even when $t / t_{a}$ is large, and an ACTRW treatment for dynamics in this regime will be essential.

\section{Proof of asymptotic behavior for one dimension}

We now prove the validity of Eq. (30) using a method developed in Ref. 53. The main idea is to show that moments of the ACTRW, are in the asymptotic limit described well by Eq. (30). For simplicity we assume a one-dimensional symmetric random walk.

The moment generating function $f(\mathbf{k})$ is expanded

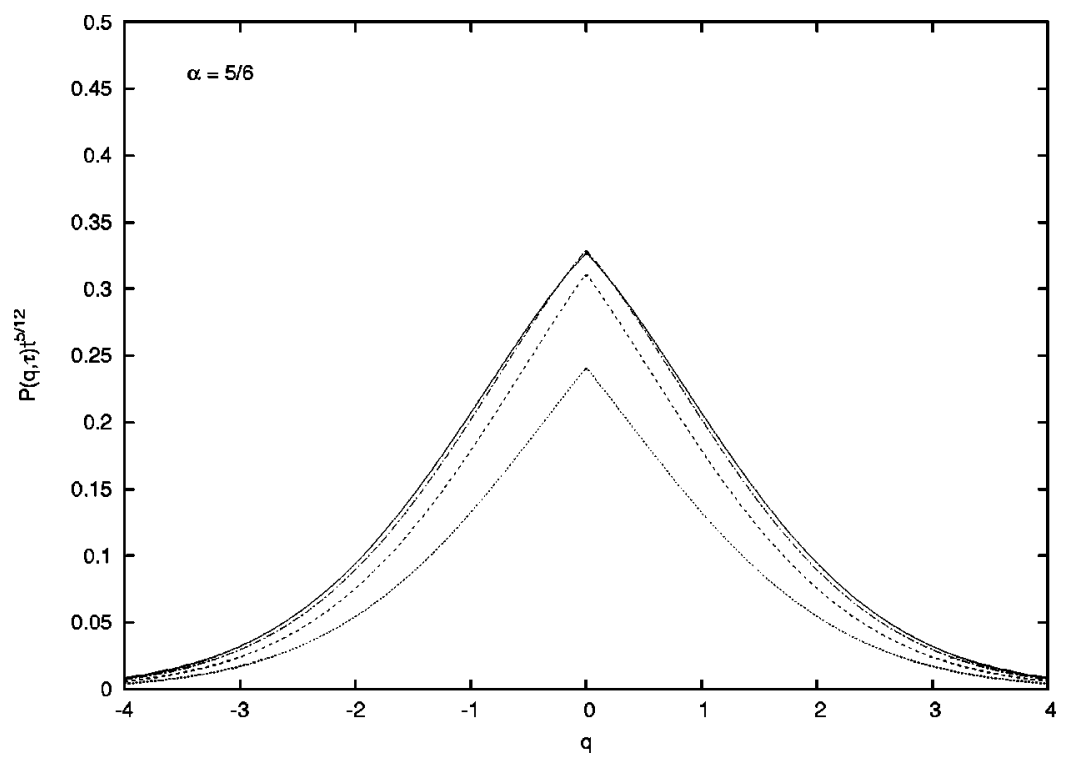

FIG. 5. Same as Fig. 3 for $\alpha=5 / 6$. 


$$
f(\mathbf{k})=1-m_{2} \frac{k^{2}}{2}+m_{4} \frac{k^{4}}{24}-m_{6} \frac{k^{6}}{720}+\cdots .
$$

Where $m_{2}, m_{4}$, etc., are the moments of the jumps. Inserting this expansion in Eq. (12) we obtain the small $k$ expansion of the ACTRW moment generating function:

$$
\begin{aligned}
P(k, s, u)= & \frac{1}{s u}-\frac{h_{s}(u)}{u}\left\{[1+\Omega(u)] m_{2} \frac{k^{2}}{2}\right. \\
& -[1+\Omega(u)]\left(m_{4}+6 \Omega(u) m_{2}^{2}\right) \frac{k^{4}}{24} \\
& +[1+\Omega(u)]\left[m_{6}-30 \Omega(u) m_{2} m_{4}\right. \\
& \left.+90 \Omega^{2}(u) m_{2}^{3}\right] \frac{k^{6}}{720}+\cdots,
\end{aligned}
$$

where

$$
\Omega(u)=\psi(u) /[1-\psi(u)] .
$$

The moments $\left\langle x^{n}(s, u)\right\rangle$ of the ACTRW are defined in the usual way

$$
P(k, s, u)=\sum_{n=0}^{\infty}\left\langle x^{n}(s, u)\right\rangle \frac{(i k)^{n}}{n !} .
$$

Comparing Eq. (39) with Eq. (41) we have

$$
\left\langle x^{0}\right\rangle=\frac{1}{s u},
$$

which means that normalization is conserved. For the second moment we obtain

$$
\left\langle x^{2}(s, u)\right\rangle=\frac{h_{s}(u)}{u}[1+\Omega(u)]
$$

which is the same as Eq. (17). The fourth moment is more interesting,

$$
\left\langle x^{4}(s, u)\right\rangle=\frac{h_{s}(u)}{u}[1+\Omega(u)]\left[m_{4}+6 \Omega(u) m_{2}^{2}\right] .
$$

Higher order moments are obtained in a similar way, for the sake of space they are not included here. Odd moments vanish due to the symmetry of the random walk.

One can easily see that the ACTRW $n$th moment $\left\langle x^{n}(s, u)\right\rangle$ depends on the microscopic jump moments $\left\{m_{2}, \cdots, m_{n}\right\}$. However, in the limit $u \rightarrow 0$,

$$
\left\langle x^{n}(s, u)\right\rangle \sim \frac{h_{s}(u)}{u} \frac{1}{\left(A u^{\alpha}\right)^{n / 2}} m_{2}^{n / 2} \frac{n !}{2^{n / 2}},
$$

which depends on $m_{2}$ but not on the higher order jump moments $m_{4}, m_{6}$, etc. Thus, the moments $m_{n}$ with $n>2$ are the irrelevant parameters in this problem. Inserting Eq. (45) in Eq. (41) we have

$$
P(k, u, s) \sim \sum_{n=0}^{\infty} \frac{h_{s}(u)}{u}(i k)^{n}\left(\frac{m_{2}}{2 A u^{\alpha}}\right)^{n / 2} .
$$

Since we are interested in the limit where $t$ and $t_{a}$ are large, the ratio $t / t_{a}$ being arbitrary, the corresponding Laplace variables $u$ and $s$ must approach zero their ratio being arbitrary. Therefore $h_{s}(u)$ in Eq. (46) is given by its asymptotic form in Eq. (8). Inserting this expression for $h_{s}(u)$ into Eq. (46), setting $m_{2}=1$, and then summing over $n$, we find an expression for $P(k, u, s)$ that is the same as Eq. (29). Equation (29) when transformed yields Eq. (30). To conclude we showed that Eq. (29) describes the small $s, u$ behavior of the ACTRW moments, hence it follows that Eq. (30) describes the long time $t$ and $t_{a}$ behavior of the ACTRW Green function, the set of moments $m_{4}, m_{6}$, etc., are unimportant in this limit.

\section{E. Behavior on the origin}

Using Eq. (33) we investigate the nonsingular part of the ACTRW on the origin. For $d=1$, we find

$$
\left.P\left(x, t_{a}, t\right)\right|_{x=0}=t^{-\alpha / 2} g\left(\frac{t}{t_{a}}\right),
$$

where

$$
g(z)=z^{\alpha / 2} \frac{\sin (\pi \alpha)}{2 \pi \Gamma(1-\alpha / 2)} \int_{0}^{z} \mathrm{~d} y \frac{(z-y)^{-\alpha / 2}}{(1+y) y^{\alpha}} .
$$

Hence

$$
\left.P\left(x, t_{a}, t\right)\right|_{x=0} \sim \begin{cases}\frac{t^{-\alpha / 2}}{2 \Gamma(\alpha) \Gamma(2-3 \alpha / 2)}\left(\frac{t}{t_{a}}\right)^{1-\alpha}, & t \ll t_{a}, \\ \frac{t^{-\alpha / 2}}{2 \Gamma(1-\alpha / 2)}, & t \gg t_{a} .\end{cases}
$$

In the limit $t \gg t_{a}$ we recover standard CTRW behavior. ${ }^{5}$

In Fig. 6, we present the behavior of the ACTRW on the origin. The ratio of the scaled nonsingular ACTRW Green function to the Montroll-Weiss nonequilibrium CTRW Green function on the origin, $\left.P\left(x, t, t_{a}\right) t^{\alpha / 2}\right|_{x=0} / 2 \Gamma(1$ $-\alpha / 2)$, is plotted versus the scaled dimensionless time $t / t_{a}$. This ratio approaches one in the limit $t \gg t_{a}$, showing that the ACTRW process will converge to the standard nonequilibrium CTRW behavior when $t \gg t_{a}$. It can be clearly seen in Fig. 6 that for $\alpha>1 / 2$, the ACTRW process has roughly converged to the nonequilibrium CTRW limit when $t / t_{a}$ $\simeq 1$, while for $\alpha \rightarrow 0$, the crossover to CTRW limit becomes extremely slow. For example, when $\alpha=1 / 12$, large deviations from the CTRW limit are clearly observed even when $t / t_{a}=10^{8}$. Since the limit $\alpha \rightarrow 0$ is important for several systems, ${ }^{19,20,54}$ it becomes clear that when $\alpha$ is small, the convergence towards the standard CTRW results becomes extremely slow, and the aging effect is of importance even when $t>t_{a}$.

From Eq. (49), we see that for $t \ll t_{a}$ the nonsingular part of $\left.P\left(x, t_{a}, t\right)\right|_{x=0}$ increases with time $t$ when $\alpha<2 / 3$. This unusual behavior is not unphysical, because the singular delta function term is a decreasing function of time, and the total probability of finding the random walker in a small vicinity of the origin is decreasing monotonically with time as expected.

\section{BIASED ACTRW}

We now consider one-dimensional biased ACTRW. We therefore use the small $k$ expansion of $f(k)$ : 


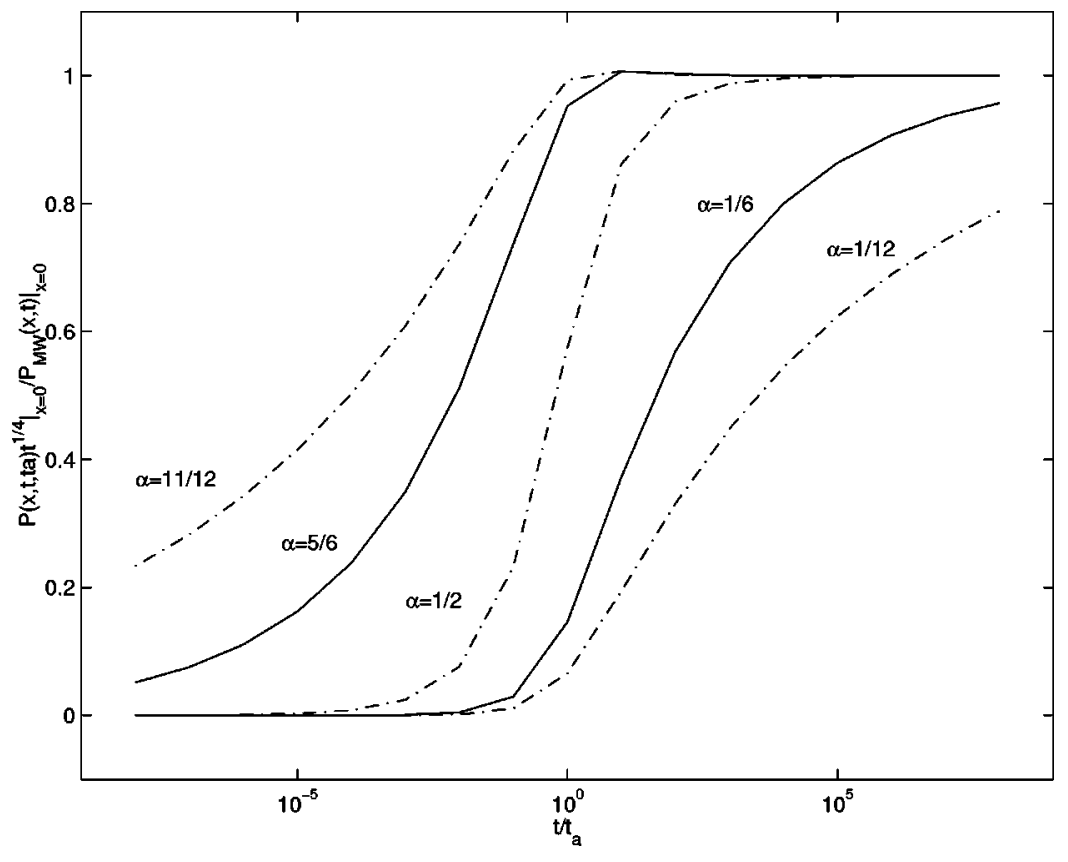

FIG. 6. The behavior of the nonsingular Green function on the origin normalized by the long time solution of the Montroll-Weiss nonequilibrium CTRW. The convergence of ACTRW towards the CTRW result is extremely slow when $\alpha \rightarrow 0$.

$$
f(k)=1+i k m_{1}-\frac{k^{2}}{2} m_{2} \cdots,
$$

where $m_{1}>0$ is the averaged jump length. Differentiating Eq. (12) once with respect to $k$ and taking $k=0$ we find the mean displacement of the random walker in $s, u$ space,

$$
\langle x(s, u)\rangle=\frac{m_{1} h_{s}(u)}{u[1-\psi(u)]},
$$

where $h_{s}(u)$ is defined in Eq. (3). Differentiating Eq. (12) twice with respect to $k$, we find the second moment of the biased random walk

$\left\langle x^{2}(s, u)\right\rangle=\frac{h_{s}(u)}{u[1-\psi(u)]}\left[2 m_{1}^{2} \frac{\psi(u)}{1-\psi(u)}+m_{2}\right]$.

\section{A. Aging Einstein relation}

We now derive a relation between the mean square displacement in the absence of bias, and the mean displacement of the particle in the presence of bias, reflecting the fluctuation-dissipation relation valid within linear response theory (see Refs. 38, 55-58 for related work). The case of zero aging $t_{a}=0$ was discussed in Ref. 59, where some conceptual problems of linear response theory for systems exhibiting anomalous type of diffusion was discussed (e.g., the nonstationarity of the process, the dependence of $\alpha$ on external field).

We assume that the random walk is on a onedimensional lattice with lattice spacing $a$, therefore

$$
f(x)=P_{L} \delta(x-a)+P_{R} \delta(x+a) .
$$

Here $P_{L}+P_{R}=1$, hence the jump moments in Eq. (50) are $m_{1}=\left(P_{R}-P_{L}\right) a$ and $m_{2}=a^{2}$. We assume that the process obeys local detailed balance, namely $P_{L} / P_{R}$ $=\exp \left(-a F / k_{b} T\right)$, where $T$ is the temperature. Using these conditions, and the assumption of weak field $a F / k_{b} T \ll 1$, we have $m_{1} \simeq a^{2} F / 2 k_{b} T$. Using Eqs. (17) and (51), we find

$$
\langle x(s, u)\rangle_{F}=\frac{F}{2 k_{b} T}\left\langle x^{2}(s, u)\right\rangle_{0} .
$$

The subscript $F$ in Eq. (54) indicates the presence of external field $F .\left\langle x^{2}(s, u)\right\rangle_{0}$ is the mean square displacement in the absence of a field, i.e., Eq. (52) with $m_{1}=0$ and $m_{2}=a^{2}$. Since the equation holds for the $s, u$ domain, it holds also for the $t_{a}, t$ domain

$$
\left\langle x\left(t_{a}, t\right)\right\rangle_{F}=\frac{F}{2 k_{b} T}\left\langle x^{2}\left(t_{a}, t\right)\right\rangle_{0} .
$$

Thus the mean square displacement of the particle in the absence of the field (the fluctuation) yields the mean displacement in the presence of a weak field. When the waiting times are exponentially distributed, we obtain the usual Einstein relation between mobility and diffusion constant, which is independent of the age of the process $t_{a}$. For experimental verification of Eq. (54) in the nonaging regime $t_{a}=0$ and with $\alpha<1$, see Refs. 60-62.

\section{B. Asymptotic behavior of biased ACTRW}

From Eq. (51) we can derive the behavior of the mean displacement in exactly the same way as done in Sec. IV A, and find

$$
\left\langle x\left(t_{a}, t\right)\right\rangle \sim \begin{cases}\frac{m_{1} t^{\alpha}}{A \Gamma(1+\alpha)}, & t \gg t_{a} \\ \frac{m_{1} t t_{a}^{\alpha-1}}{A \Gamma(\alpha)}, & t \ll t_{a} .\end{cases}
$$

For the second moment we use the small $s, u$ behavior of Eq. (52) and find

$$
\left\langle x^{2}(s, u)\right\rangle \sim \frac{1}{A u^{1+\alpha}} \frac{u^{\alpha}-s^{\alpha}}{s^{\alpha}(u-s)}\left[\frac{2 m_{1}^{2}}{u^{\alpha} A}+m_{2}\right] .
$$


Inverting this equation using Eq. (56), we investigate now the dispersion

$$
\sigma\left(t_{a}, t\right) \sim \sqrt{\left\langle x^{2}\left(t_{a}, t\right)\right\rangle-\left\langle x\left(t_{a}, t\right)\right\rangle^{2}} .
$$

Considering first the $u \ll s$ limit corresponding to $t \gg t_{a}$ we recover Shlesinger's result ${ }^{63}$

$\sigma\left(t_{a}, t\right) \sim \sqrt{\frac{m_{1}^{2} t^{2 \alpha}}{A^{2}}\left[\frac{2}{\Gamma(1+2 \alpha)}-\frac{1}{\Gamma^{2}(1+\alpha)}\right]+\frac{m_{2} t^{\alpha}}{A \Gamma(1+\alpha)}}$,

hence if $m_{1} \neq 0$ and $\alpha<1$, one finds

$\sigma\left(t_{a}, t\right) \sim \frac{m_{1} t^{\alpha}}{A} \sqrt{\frac{2}{\Gamma(1+2 \alpha)}-\frac{1}{\Gamma^{2}(1+\alpha)}}$.

For $t \gg t_{a}$ the dispersion of the biased CTRW grows like the mean Eq. (56), a behavior very different than normal Gaussian diffusion.

Considering the $s \ll u$ limit of Eq. (57) and using Eq. (56), we find for aging limit $t \ll t_{a}$

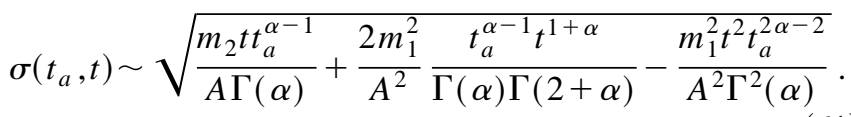

Hence if $m_{1} \neq 0$ and $\alpha<1$, one finds

$\sigma\left(t_{a}, t\right) \sim \frac{m_{1}}{A} \sqrt{\frac{2}{\Gamma(\alpha) \Gamma(2+\alpha)}} \frac{t^{(1+\alpha) / 2}}{t_{a}^{(1-\alpha) / 2}}$,

which is valid for $t_{a} \gg t \gg A^{1 / \alpha}$. As expected the dispersion decreases as age of the processes becomes older. Note that as $\alpha \rightarrow 0$, the first term in Eq. (61) becomes important.

For $\alpha=1$ one finds

$$
\sigma(t) \sim \sqrt{\frac{m_{2} t}{A}},
$$

where in this case $A$ has the meaning of the mean time between jumps. The dispersion in this case is independent of the age of the system $t_{a}$, as expected from normal diffusion. We see that the dispersion in normal diffusion processes is controlled by the second moment of jump lengths $m_{2}$ (even when $m_{1} \neq 0$ ), while for CTRW and ACTRW $m_{1} \neq 0$ is the relevant parameter.

\section{POSSIBLE APPLICATION: SCHER-MONTROLL TRANSPORT}

In this section we briefly point out one possible application of ACTRW. Scher and Montroll ${ }^{7}$ modeled transport in disordered medium based on CTRW theory. The fundamental reasons of why and when their approach is valid, while being the subject of much theoretical research, ${ }^{64,65}$ are not totally solved. What is clear is that on a phenomenological level, one can use the Scher-Montroll approach to fit behaviors of charge currents in a large number of experiments in very different systems. For example, transport of charge carriers in organic photorefractive glasses, ${ }^{66}$ nanocrystalline $\mathrm{T}_{\mathrm{i}} \mathrm{O}_{2}$ electrodes, ${ }^{67}$ conjugated polymer system poly $p$-phenylene, ${ }^{68,69}$ and liquid crystalline xinc octakis. ${ }^{70}$

Scher and Montroll model such transport processes using nonequilibrium biased CTRW theory with an effective waiting time distribution. In experiments, this corresponds to charge transport which is started at time $t=0$, for example, by a short photo flash applied on the system. After the initial triggering of the process, the charge carriers are transported using an external bias. For such initial conditions, we know that it is useful to assume that the physical transport process is described by the nonequilibrium biased CTRW.

In an aging experiment one would start the process, by an external impulse (e.g., a photo flash), then wait for an aging period $t_{a}$, and only after that period add the external bias. In this case, biased aging CTRW might become a useful tool describing the aging transport. At this time it is still an open question if ACTRWs can be used to describe aging in real systems. Further it is not clear if aging in the abovementioned systems ${ }^{66-70}$ is measurable, and if so do these very different systems exhibit any common aging effects in their transport?

In the nonaging experiments ${ }^{66-70}$ one measures the current of charge carriers, which according to the predictions of Scher and Montroll exhibit a universal behavior: $I(t) \propto t^{\alpha-1}$ for short times and $I(t) \propto t^{-\alpha-1}$ for long times. The transition time $t_{L}$ between these two behaviors depends among other things on the length of the system. The short time behavior corresponds to $I(t) \propto(\mathrm{d} / \mathrm{d} t)\langle x\rangle$, with the nonequilibrium CTRW behavior $\langle x\rangle \propto t^{\alpha}$, which yields immediately $I(t)$ $\propto t^{\alpha-1}$. The long time behavior is more complicated, and is due to absorbing boundary condition.

In this paper we have calculated the mean displacement of the biased ACTRW, without including the influence of the boundary. Thus we provide the aging corrections to the short time behavior of Scher-Montroll transport. According to Eq. (56), the Scher-Montroll behavior $I(t) \propto t^{\alpha-1}$ is replaced with $I(t) \propto t_{a}^{\alpha-1}$ when $t \ll t_{a} \ll t_{L}$. This behavior is independent of $t$, similar to behavior of normal currents. In this aging regime, the current decreases as $t_{a}$ is increased, while in the nonaging case the current is decreasing when the forward time $t$ is increased. The assumption made is that the dispersion of the probability packet during the aging period, is small compared with the length of the system. A detailed investigation of aging in Scher-Montroll transport systems will be given elsewhere.

\section{SUMMARY}

We have derived an exact expression for the Green function of ACTRW in Fourier-double Laplace space. This generalized Montroll-Weiss equation describes dynamics of a large class of random walks. Since the CTRW describes a large class of physical and chemical systems, mainly disordered systems, we expect that ACTRW will be a valuable tool when aging effects are investigated in these systems. Interesting aging behaviors are found when the system turns nonergodic, namely when the mean waiting time diverges, $\alpha<1$. We note that also when the mean waiting time is finite, aging behaviors may be observed, however only within a certain time window.

In many cases anomalous diffusion is characterized based on an anomalous behavior of the mean square displacement $\left\langle x^{2}\right\rangle \sim t^{\alpha}$. However, for nonbiased ACTRWs we 
showed that when $t \ll t_{a}\left\langle x^{2}\right\rangle \sim t / t_{a}^{1-\alpha}$, hence the random walk exhibits a normal behavior with respect to forward time $t$. Thus if one measures the displacement of CTRW particles in time interval $(0, t)$, for some fixed $t_{a}$, and considers the mean square displacement, one may reach the wrong conclusion that the anomalous process is normal. The anomalous behavior, with respect to the forward time $t$, is more easily observed in the behavior of the Green function, or $p_{0}\left(t_{a}, t\right)$.

We showed that asymptotic behavior of the Green function is related to a few parameters of the underlying walk $\alpha, A$, and $m_{2}$, while other informations contained in $\psi(t)$ and $f(\mathbf{r})$ are irrelevant. The Green function behavior is nonGaussian when $\alpha<1$, it is related to Lévy's generalized central limit theorem and to Dynkin's limit theorem. Unlike standard random walks or nonequilibrium CTRWs, the asymptotic Green function is a sum of two terms: a singular term corresponding to random walks where number of jumps is zero and a nonsingular term corresponding to random walks where number of jumps is one or more. Finally, we note that the fractional Fokker-Planck equation framework, ${ }^{2,39,71}$ developed based on CTRW concepts, ${ }^{42,53}$ can be modified based on the results obtained in this paper to include aging effects. The fractional kinetic equations describing ACTRWs will be a subject for a future publication.

\section{ACKNOWLEDGMENTS}

One of the authors (E.B.) thanks J. P. Bouchaud for pointing out Ref. 29. Discussions with L. Levitov and A. Heuer motivated this work.

\section{APPENDIX: DERIVATION OF EQ. (3)}

In this Appendix, we derive Eq. (3) using a method which is slightly different then the one used in Ref. 29. Consider a nonequilibrium renewal process which starts at time $t=0$ (in the ACTRW this initial time is $-t_{a}$ ). Let $\bar{t}_{i} i$ $=1, \ldots, N$, be dots on the time axis on which jumping events occur. Let $\bar{t}_{N+1}$ denote the time on which the first jump event occurred which is larger than $t_{a}$, namely $\bar{t}_{N}$ $<t_{a}<\bar{t}_{N+1}$. Note that $N$ itself is not fixed, it is a random variable. The time intervals between jumps events are denoted by $\tau_{i} \equiv \bar{t}_{i+1}-\bar{t}_{i}$.

The random variable we are interested in is $t_{1}$, where $t_{1} \equiv \bar{t}_{N+1}-t_{a}$. In the context of renewal theory $t_{1}$ is called residual waiting time. ${ }^{28}$ Since $t_{a}$ is a parameter in the problem, knowledge of statistical properties of $\bar{t}_{N+1}$ yields the distribution of $t_{1}$. Hence let $P_{t_{a}}\left(\bar{t}_{N+1}\right)$ denote the PDF of the random variable $\bar{t}_{N+1}$. It is given by

$$
P_{t_{a}}\left(\bar{t}_{N+1}\right)=\sum_{N=0}^{\infty}\left\langle\delta\left(\bar{t}_{N+1}-\sum_{i=1}^{N+1} \tau_{i}\right) I\left(\bar{t}_{N}<t_{a}<\bar{t}_{N+1}\right)\right\rangle_{N} .
$$

Here $I\left(\bar{t}_{N}<t_{a}<\bar{t}_{N+1}\right)=1$ if $\bar{t}_{N}<t_{a}<\bar{t}_{N+1}$, otherwise it is zero. The average in Eq. (A1) is

$$
\langle\cdots\rangle_{N}=\left\langle\Pi_{i=1}^{N+1} \int_{0}^{\infty} \psi\left(\tau_{i}\right) \mathrm{d} \tau_{i} \cdots\right\rangle .
$$

We consider the double Laplace transform $t_{a} \rightarrow s$ and $\bar{t}_{N+1} \rightarrow u \quad$ of $P_{t_{a}}\left(\bar{t}_{N+1}\right) \quad$ Eq. (A1), $P_{s}(u)$. Using $\bar{t}_{N}$ $=\sum_{i=1}^{N} \tau_{i}$, we have

$$
\begin{aligned}
\int_{0}^{\infty} e^{-t_{a} s} I\left(\bar{t}_{N}<t_{a}<\bar{t}_{N+1}\right) \mathrm{d} t_{a} \\
=\frac{e^{-\bar{t}_{N} s}-e^{-\bar{t}_{N+1} s}}{s}=\frac{e^{-s \Sigma_{i=1}^{N} \tau_{i}-e^{-s \Sigma_{i=1}^{N+1} \tau_{i}}}}{s},
\end{aligned}
$$

and

$$
\int_{0}^{\infty} e^{-u \bar{t}_{N+1}} \delta\left(\bar{t}_{N+1}-\sum_{i=1}^{N+1} \tau_{i}\right) \mathrm{d} \bar{t}_{N+1}=e^{-u \Sigma_{i=1}^{N+1} \tau_{i}} .
$$

Using Eqs. (A3) and (A4), we have from Eq. (A1),

$$
\begin{aligned}
P_{s}(u)= & \sum_{N=0}^{\infty}\left\langle\exp \left(-u \sum_{i=1}^{N+1} \tau_{i}\right)\right. \\
& \left.\times \frac{\exp \left(-s \Sigma_{i=1}^{N} \tau_{i}\right)-\exp \left(-s \Sigma_{i=1}^{N+1} \tau_{i}\right)}{s}\right\rangle .
\end{aligned}
$$

Using the fact that the random variables $\tau_{i}$ are independent and identically distributed, we have

$$
P_{s}(u)=\frac{1}{s} \sum_{N=0}^{\infty}\left[\psi^{N}(u+s) \psi(u)-\psi^{N+1}(u+s)\right],
$$

where $\psi(u+s)=\int_{0}^{\infty} \exp [-(u+s) \tau] \psi(\tau) \mathrm{d} \tau$. Summing Eq. (A6) we find

$$
P_{s}(u)=\frac{\psi(u)-\psi(u+s)}{s} \frac{1}{1-\psi(u+s)} .
$$

Now the PDF $h_{t_{a}}\left(t_{1}\right)$ is obtained from $P_{t_{a}}\left(\bar{t}_{N+1}\right)$ using $t_{1}=\bar{t}_{N+1}-t_{a}$. According to definition of Laplace transform, we can write

$$
h_{t_{a}}\left(t_{1}\right)=\mathcal{L}_{u \rightarrow t_{1}, s \rightarrow t_{a}}^{-1}\left\{\int_{0}^{\infty} \mathrm{d} t_{a} e^{-s t_{a}} \int_{0}^{\infty} \mathrm{d} t_{1} e^{-u t_{1}} h_{t_{a}}\left(t_{1}\right)\right\},
$$

where $\mathcal{L}^{-1}$ is the double inverse Laplace transform. We use $t_{1}=\bar{t}_{N+1}-t_{a}$ and find

$$
\begin{aligned}
h_{t_{a}}\left(t_{1}\right)= & \mathcal{L}_{u \rightarrow t_{1}, s \rightarrow t_{a}}^{-1}\left\{\int_{0}^{\infty} \mathrm{d} t_{a} e^{-s t_{a}} \int_{t_{a}}^{\infty} \mathrm{d} t_{1} e^{-u\left(\bar{t}_{N+1}-t_{a}\right)}\right. \\
& \left.\times P_{t_{a}}\left(\bar{t}_{N+1}\right)\right\} .
\end{aligned}
$$

Hence it is easy to see that

$$
h_{s}(u)=P_{s-u}(u) .
$$

Inserting Eq. (A7) in Eq. (A10), we find Eq. (3).

Remark: The time $t_{a}-\bar{t}_{N}$ is called the spent time, ${ }^{28}$ and in the limit of long $t_{a}$ Feller shows that this time is distributed according to the arc-sine law.

\footnotetext{
${ }^{1}$ J.-P. Bouchaud and A. Georges, Phys. Rep. 195, 127 (1990).

${ }^{2}$ R. Metzler and J. Klafter, Phys. Rep. 339, 1 (2000).

${ }^{3}$ S. Havlin and D. Ben Avraham, Adv. Phys. 51, 187 (2002).

${ }^{4}$ E. W. Montroll and G. H. Weiss, J. Math. Phys. 6, 167 (1965).
} 
${ }^{5}$ G. H. Weiss, Aspects and Applications of the Random Walk (NorthHolland, Amsterdam, New York, Oxford, 1994).

${ }^{6}$ J. W. Haus and K. W. Kehr, Phys. Rep. 150, 263 (1987).

${ }^{7}$ H. Scher and E. Montroll, Phys. Rev. B 12, 2455 (1975).

${ }^{8}$ J. D. Bryngelson and P. G. Wolynes, J. Phys. Chem. 93, 6902 (1989).

${ }^{9}$ S. S. Plotkin and P. G. Wolynes, Phys. Rev. Lett. 80, 5015 (1998).

${ }^{10}$ C. L. Lee, G. Stell, and J. Wang, J. Chem. Phys. 118, 959 (2003).

${ }^{11}$ J. Klafter, M. F. Shlesinger, and G. Zumofen, Phys. Today 49, 33 (1996).

${ }^{12}$ T. H. Solomon, E. R. Weeks, and H. L. Swinney, Phys. Rev. Lett. 71, 3975 (1993).

${ }^{13}$ A. I. Saichev and G. M. Zaslavsky, Chaos 7, 753 (1997).

${ }^{14}$ G. M. Zaslavsky, Phys. Rep. 371, 461 (2002).

${ }^{15}$ C. F. Vardeman and J. D. Gezelter, J. Phys. Chem. A 105, 2568 (2001).

${ }^{16}$ J. Sung, E. Barkai, R. Silbey, and S. Lee, J. Chem. Phys. 116, 2338 (2002).

${ }^{17}$ Y. Jung, E. Barkai, and R. Silbey, Chem. Phys. 284, 181 (2002).

${ }^{18}$ M. Feigelman and V. M. Vinokur, J. Phys. (France) 49, 1731 (1988).

${ }^{19}$ E. Marinari and G. Parisi, J. Phys. A 26, L1149 (1993).

${ }^{20}$ P. Le Doussal, C. Monthus, and D. S. Fisher, Phys. Rev. E 59, 4795 (1999).

${ }^{21}$ L. Laloux and P. Le Doussal, Phys. Rev. E 57, 6296 (1998).

${ }^{22}$ I. M. Sokolov, A. Blumen, and J. Klafter, Physica A 302, 268 (2001).

${ }^{23}$ P. Allegrini et al., Phys. Rev. E 66, 015101(R) (2002).

${ }^{24} \mathrm{X}$. Brokmann et al., cond-mat/0211171.

${ }^{25}$ L. Berthier et al., cond-mat/0211106.

${ }^{26}$ C. Monthus and J. P. Bouchaud, J. Phys. A 29, 3847 (1996).

${ }^{27}$ E. B. Dynkin, Selected Translations in Mathematical Statistics and Probability (American Mathematical Society, Providence, 1961), Vol. 1, p. 249.

${ }^{28} \mathrm{~W}$. Feller, An Introduction to Probability Theory and its Application (Wiley, New York, 1970), Vol. 2, Chaps. 13 and 14.

${ }^{29}$ C. Gordeche and J. M. Luck, J. Stat. Phys. 104, 489 (2001).

${ }^{30}$ B. Rinn, P. Maass, and J. P. Bouchaud, Phys. Rev. Lett. 84, 5403 (2000).

${ }^{31}$ P. Sollich, F. Lequeux, P. Hebraud, and M. E. Cates, Phys. Rev. Lett. 78, 2020 (1997)

${ }^{32}$ R. M. L. Evans, M. E. Cates, and P. Sollich, Eur. Phys. J. B 10, 705 (1999).

${ }^{33}$ B. Doliwa and A. Heuer, cond-mat/0205283.

${ }^{34}$ R. A. Denny, D. R. Reichmann, and J. P. Bouchaud, Phys. Rev. Lett. 90, 025503 (2003).

${ }^{35}$ E. Barkai, Phys. Rev. Lett. (to be published).

${ }^{36}$ D. A. Stariolo, Phys. Rev. E 55, 4806 (1997).
${ }^{37}$ N. Pottier, Physica A. 317, 371 (2002).

${ }^{38}$ N. Pottier and A. Mauger, Physica A 282, 77 (2000).

${ }^{39}$ R. Metzler, E. Barkai, and J. Klafter, Phys. Rev. Lett. 82, 3563 (1999).

${ }^{40}$ E. Barkai, Phys. Rev. E 63, 046118 (2001).

${ }^{41}$ I. M. Sokolov, J. Klafter, and A. Blumen, Phys. Today 55, 48 (2002).

${ }^{42}$ E. Barkai, R. Metzler, and J. Klafter, Phys. Rev. E 61, 132 (2000).

${ }^{43}$ I. M. Sokolov, A. Blumen, and J. Klafter, Europhys. Lett. 56, 175 (2001).

${ }^{44}$ J. K. E. Tunaley, Phys. Rev. Lett. 33, 1037 (1974).

${ }^{45}$ E. Barkai, V. Fleurov, and J. Klafter, Phys. Rev. E 61, 1164 (2000).

${ }^{46}$ A. I. Shushin, J. Chem. Phys. 114, 4883 (2001).

${ }^{47}$ The main mathematical (Ref. 28) tool in investigating asymptotic behavior of ACTRW will be the Tauberian theorem: if $g(u) \sim u^{-\theta}$ as $u \rightarrow 0$, with $\theta>0$ then $g(t) \sim t^{\theta-1} / \Gamma(\theta)$ as $t \rightarrow \infty$ where $g(u)$ and $g(t)$ are Laplace pairs.

${ }^{48}$ R. Hilfer and L. Anton, Phys. Rev. E 51, R848 (1995).

${ }^{49}$ A. Erdelyi et al., Higher Transcendental Functions (Krieger, Malabar, 1981), Vol. 3.

${ }^{50}$ W. R. Schneider and W. Wyss, J. Math. Phys. 30, 134 (1989).

${ }^{51}$ L. D'Amore et al., ACM Trans. Math. Softw. 25, 279 (1999).

${ }^{52}$ L. D'Amore et al., ACM Trans. Math. Softw. 25, 306 (1999).

${ }^{53}$ E. Barkai, Chem. Phys. 284, 13 (2002).

${ }^{54}$ J. Drager and J. Klafter, Phys. Rev. Lett. 84, 5998 (2000).

${ }^{55}$ T. S. Grieg and N. E. Israeloff, Phys. Rev. Lett. 83, 5038 (1999).

${ }^{56}$ D. Harrison and M. Socio, Phys. Rev. Lett. 88, 257202 (2002).

${ }^{57}$ R. Mercado et al., Phys. Rev. Lett. 89, 100601 (2002).

${ }^{58}$ O. Benichou and G. Oshanin, Phys. Rev. E 66, 031101 (2002).

${ }^{59}$ E. Barkai and V. Fleurov, Phys. Rev. E 58, 1296 (1998).

${ }^{60}$ Q. Gu et al., Phys. Rev. Lett. 76, 3196 (1996).

${ }^{61}$ F. Amblard et al., Phys. Rev. Lett. 77, 4470 (1996).

${ }^{62}$ E. Barkai and J. Klafter, Phys. Rev. Lett. 81, 1134 (1998).

${ }^{63}$ M. F. Shlesinger, J. Stat. Phys. 10, 421 (1974).

${ }^{64}$ G. Pfister and H. Scher, Adv. Phys. 27, 747 (1978).

${ }^{65}$ A. V. Lopatin and V. M. Vinokur, Phys. Rev. Lett. 86, 1817 (2001).

${ }^{66}$ M. Grasruck et al., Phys. Rev. B 60, 16543 (1999).

${ }^{67}$ J. Nelson, Phys. Rev. B 59, 15374 (1999).

${ }^{68}$ P. W. M. Bloom and M. C. J. M. Vissenberg, Phys. Rev. Lett. 80, 3819 (1998).

${ }^{69}$ S. S. Pandey et al., Jpn. J. Appl. Phys., Part 1 40, 5350 (2001).

${ }^{70}$ Y. Yuan, B. A. Greg, and M. F. Lawrence, J. Mater. Res. 15, 2494 (2000).

${ }^{71}$ E. Barkai and R. Silbey, J. Phys. Chem. B 104, 3866 (2000). 